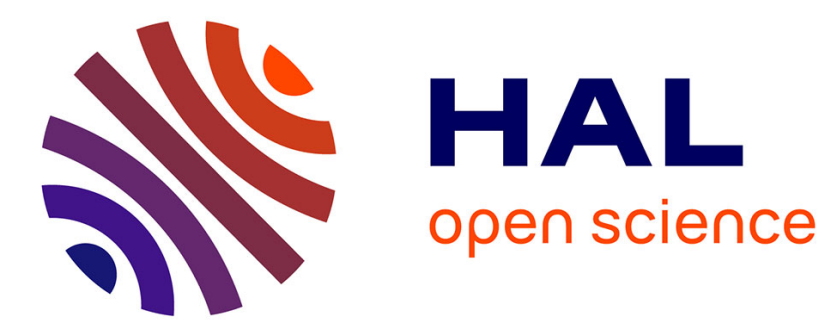

\title{
Automatic sensitivity analysis of a finite volume model for two-dimensionnal shallow water flows
}

\author{
O. Souhar, J.B. Faure, André Paquier
}

\section{To cite this version:}

O. Souhar, J.B. Faure, André Paquier. Automatic sensitivity analysis of a finite volume model for two-dimensionnal shallow water flows. Environmental Fluid Mechanics, 2007, 7, p. 303 - p. 315. 10.1007/s10652-007-9028-5 . hal-00453843

\section{HAL Id: hal-00453843 \\ https://hal.science/hal-00453843}

Submitted on 5 Feb 2010

HAL is a multi-disciplinary open access archive for the deposit and dissemination of scientific research documents, whether they are published or not. The documents may come from teaching and research institutions in France or abroad, or from public or private research centers.
L'archive ouverte pluridisciplinaire HAL, est destinée au dépôt et à la diffusion de documents scientifiques de niveau recherche, publiés ou non, émanant des établissements d'enseignement et de recherche français ou étrangers, des laboratoires publics ou privés. 
Author-produced version of the article published in Environnemental Fluid Mechanics, 2007, 7, 303-315.

The original publication is available at http://www.springerlink.com/ doi : 10.1007/s10652-007-9028-5 


\title{
Automatic sensitivity analysis of a finite volume model for two-dimensional shallow water flows
}

\author{
Souhar, O. ${ }^{1}$, Faure, J-B. ${ }^{2}$ And Paquier, A. ${ }^{3}$ \\ June 21, 2007 \\ Cemagref de Lyon \\ Hydrology-Hydraulic research unit \\ 3 bis, quai Chauveau-CP 220, \\ 69336 Lyon Cedex 09, FRANCE \\ Tel. $+33472208787-$ Fax. +33478477875
}

${ }^{1}$ Corresponding author, E-mail: drsouhar@hotmail.com

2 faure@lyon.cemagref.fr

3 paquier@lyon.cemagref.fr

\begin{abstract}
Given a numerical model for solving two-dimensional Shallow Water Equations, we are interested in the robustness of the simulation by identifying the rate of change of the water depths and discharges with respect to a change in the bottom friction coefficients. Such a sensitivity analysis can be carried out by computing the corresponding derivatives. Automatic Differentiation (AD) is an efficient numerical method, free of approximation errors, to evaluate derivatives of the objective function specified by the computer program, Rubar20 for example. In this paper AD software tool Tapenade is used to compute forward derivatives. Numerical tests were done to show the robustness of the model and to demonstrate the efficiency of these $\mathrm{AD}$-derivatives.
\end{abstract}

Key words : Automatic differentiation, Hydraulic model, Sensitivity analysis, Uncertainty propagation, Steady flow. 


\section{Introduction}

The two-dimensional Shallow Water Equations are a relevant basis for modelling free surface flows, but their solution requires detailed information about the river configuration as the topographical data and the friction coefficient according to Manning law. It is also necessary to provide information bound to a particular event: these are the initial conditions of the flow as well as the upstream and downstream boundary conditions of the river.

The model input data are submitted to uncertainties difficult to evaluate and which may lead to meaningful uncertainties on the model output. The output results may be a map showing a flooded urbanized area; consequently, the induced damages may be very high. In these situations, the river can go far away from their mean water channel, submerging large areas, eventually with high velocity. In order to evaluate the uncertainties of the water level and river flow width according to the input data of a one-dimensional hydraulic model (Giraud et al. [11]), Dhervillez ([5]) and Faure et al. ([6]) tested several noising methods. Their study showed that if the bottom level is gaussian then consequently the water level is gaussian too in steady flow. On the other hand, they showed in the same paper that if the bottom level is gaussian then the river flow width is not gaussian.

In such a case, sensitivity analysis can be lead by computing partial derivatives of the output functions with respect to the corresponding parameters. The most familiar schemes to approximate derivatives of functions specified by computer programs are the central differences and the so-called forward finite differences. So for a smooth function $F: \mathbb{R}^{n} \longrightarrow \mathbb{R}^{m}$ and for some direction $d \in \mathbb{R}^{n}$, the central approximation, for $\bar{\varepsilon}<<1$, is defined as

$$
\nabla F(x) \cdot d \approx \frac{F(x+\bar{\varepsilon} \cdot d)-F(x-\bar{\varepsilon} \cdot d)}{2 . \bar{\varepsilon}},
$$

which have a truncation error of $\mathcal{O}\left((\bar{\varepsilon} \cdot d)^{2}\right)$. Thus, using this central operator, the gradient requires at least $(2 n)$ evaluations of the function $F$. However, the forward operator defined below requires only $(n+1)$ evaluations of $F$, but the truncation error is only of $\mathcal{O}(\bar{\varepsilon} \cdot d)$ :

$$
\nabla F(x) \cdot d \approx \frac{F(x+\bar{\varepsilon} \cdot d)-F(x)}{\bar{\varepsilon}} .
$$

From a theoretical point of view, a good accuracy of the approximate (1.1) or (1.2) is obtained for very small values of $\bar{\varepsilon}$. Nevertheless, $\bar{\varepsilon}$ may not be arbitrarily small, since for very small values, the difference between $F(x+\bar{\varepsilon} . d)$ 
and $F(x)$ is of the discretization error order, thus losing its significance. Nowadays, methods and softwares for sensitivity analysis of several engineering problems (fluid dynamics, chemistry, meteorology or environment studies etc) have demonstrated that forward sensitivities can be computed reliably and efficiently via Automatic Differentiation (AD). AD is superior to divided differences because AD-generated derivative values are free of approximation errors, see Section 4 for more details. In this paper the Tapenade software tool, developed by Tropics team INRIA Sophia-Antipolis, is used to compute the desired derivatives of the objective functions specified by the finite volume two-dimensional shallow water model Rubar20 with respect to the roughness coefficients. The output results or the objective functions are the water depth and the discharge computed by Rubar20 (Paquier [21]).

The outline of this paper is as follows. The governing equations are first presented. Details of the numerical model Rubar20 are discussed. An overview of the AD strategy is given. The numerical results are presented and the computed results are verified by comparing them with other results obtained from another hydraulic model. Finally, the sensitivity of the model according to the roughness coefficient is studied.

\section{Shallow Water Equations}

The two-dimensional shallow water equations describe the unsteady free surface flows, which simply express the conservation of mass and momentum. Usually these equations are described in terms of water depth and discharge under the following assumptions (Lai [16]): hydrostatic pressure distribution, very small bottom slopes, uniform velocity in the vertical direction and incompressible fluid. The resulting equations are

$$
\begin{aligned}
& \frac{\partial h}{\partial t}+\frac{\partial q_{x}}{\partial x}+\frac{\partial q_{y}}{\partial y}=0 \\
& \frac{\partial q_{x}}{\partial t}+\frac{\partial\left(\frac{q_{x}^{2}}{h}+g \frac{h^{2}}{2}\right)}{\partial x}+\frac{\partial\left(\frac{q_{x} q_{y}}{h}\right)}{\partial y}=-g h \frac{\partial Z}{\partial x}-g q_{x} \frac{\sqrt{q_{x}^{2}+q_{y}^{2}}}{C^{2} h^{2}}+ \\
& D\left(\frac{\partial}{\partial x}\left(h \frac{\partial\left(\frac{q x}{h}\right)}{\partial x}\right)+\frac{\partial}{\partial y}\left(h \frac{\partial\left(\frac{q x}{h}\right)}{\partial y}\right)\right) \\
& \frac{\partial q_{y}}{\partial t}+\frac{\partial\left(\frac{q_{x} q_{y}}{h}\right)}{\partial x}+\frac{\partial\left(\frac{q_{y}^{2}}{h}+g \frac{h^{2}}{2}\right)}{\partial y}=-g h \frac{\partial Z}{\partial y}-g q_{y} \frac{\sqrt{q_{x}^{2}+q_{y}^{2}}}{C^{2} h^{2}}+ \\
& D\left(\frac{\partial}{\partial x}\left(h \frac{\partial\left(\frac{q y}{h}\right)}{\partial x}\right)+\frac{\partial}{\partial y}\left(h \frac{\partial\left(\frac{q y}{h}\right)}{\partial y}\right)\right)
\end{aligned}
$$


where $t=$ time; $h=$ water depth; $Z=$ bed elevation; $q_{x}$ and $q_{y}=$ 'discharge' (water depth multiplied by velocity) along respectively the $x$-axis and the $y$-axis; $C=$ Chezy friction coefficient which is considered as a constant or equalled to $S T K \times h^{\frac{1}{6}}$ where $S T K$ is the Strickler's friction coefficient; $g=$ acceleration due to gravity; $D=$ diffusion coefficient which is estimated as a constant.

\section{Numerical model}

Based on a finite volume method, the model Rubar20 is designed to solve the two-dimensional shallow water equations on unstructured triangular and rectangular grids. The model, written in Fortran 77 has been developed at Cemagref since 1988 (Paquier [21]). It uses a Van Leer explicit projectioncorrection scheme with second-order spatial and temporal accuracy in order to avoid spurious oscillations. The two main features of the numerical scheme are described here below. In case of hydraulic structures, the classical relations for weirs, gates, ... are used instead of the shallow water equations. In fact, an hydraulic structure can be defined as a set of a few cells where fluxes through one edge are computed either from the relations for the hydraulic structure, either from an addition of a flow computed from shallow water equations and a flow coming through an hydraulic structure. This last possibility may be useful, for instance, in the case of an embankment which is submerged although some discharge crosses it through a culvert. The model has been applied to simulate different situations of flows (Mignot [17], Mignot et al. [18], Mignot et al. [19], Paquier [22], [23], Paquier and Farissier [24], Paquier and Mignot [25], Paquier et al. [26], Proust et al. [27]). The scheme is stable for Courant number less than 1 . The two main steps of the numerical scheme are described here below.

\subsection{Riemann problems}

In a finite volume approach, the updated values of the main variables $\left(h, q_{x}, q_{y}\right)$ are calculated using the fluxes crossing the edges. To estimate these fluxes, the 2-D problem constituted by the first member of the equations $(2.1)+$ $(2.2)+(2.3)$ is simplified in a 1-D problem in which the left and right values (on each side of the edge) are constant. If $x$ means the direction perpendicular 
to the edge, the simplified 1-D problem writes:

$$
\begin{gathered}
\frac{\partial h}{\partial t}+\frac{\partial q_{x}}{\partial x}=0 \\
\frac{\partial q_{x}}{\partial t}+\frac{\partial\left(\frac{q_{x}^{2}}{h}+g \frac{h^{2}}{2}\right)}{\partial x}=0 \\
\frac{\partial q_{y}}{\partial t}+\frac{\partial\left(\frac{q_{x} q_{y}}{h}\right)}{\partial x}=0
\end{gathered}
$$

The equations $(3.1)+(3.2)+(3.3)$ constitute a nonlinear hyperbolic system for the 3 -D variable $W=\left(h, q_{x}, q_{y}\right)$. Eigenvalues are $u-c, u$ and $u+c$ where $u$ is the velocity perpendicular to the edge and $c=\sqrt{g h}$ is the wave celerity. The second field is linearly degenerated whereas the first and the third ones are truly nonlinear. The Riemann invariants can be very simply computed as they respectively equal $u+2 c$ and $v, u+2 c$ and $u-2 c, u-2 c$ and $v$ where $v$ is the velocity parallel to the edge.

To solve this Riemann problem, it is possible to use a Roe type linearization (Roe [29]) which directly gives an estimate of the flux but alternative methods keeping the properties of the fields (and thus rarefactions and shocks) exist. Generally, these latter methods have the advantage to provide also an estimate of the values of the variables on the edge, which can be used when computing second member of the system $(2.1)+(2.2)+(2.3)$ (Paquier [21]). In order to obtain a second-order scheme in space, the values considered in the Riemann problem are computed by using slopes in the $\mathrm{x}$ and $\mathrm{y}$ directions (for each of the 3 variables $h, q_{x}$ and $q_{y}$ independently). For every cell, these slopes are computed by the method of the least squares (on each of the 3 variables independently) from the basis of the values at the centre of the cells which have one common edge with the given cell (Naaim [20]). The slope for any variable is later limited if the value of the given variable $A$ (which may be $h+Z, q_{x}$ or $q_{y}$ ) at the middle of one edge is not located between the value at the centre of the cell $A_{c}$ and $\alpha A_{n}+(1-\alpha) A_{c}$ where $A_{n}$ is the value at the centre of the corresponding adjacent cell. The Van Leer correction factor $\alpha$ should be selected between 0.5 and 1 .

In order to obtain second-order accuracy in time, the values of fluxes should be assessed at time $t_{n+1 / 2}=t_{n}+0.5 \Delta t$ instead of time $t_{n}$ (Paquier [21]). 


\subsection{Treatment of second member}

Using the fluxes across edges, it is possible to obtain provisional values of the variables at time $t_{n+1}=t_{n}+\Delta t$. Then we add the contribution of the second member which includes, at least, 2 terms:

i. Gravity or slope terms $\left(-g h \frac{\partial Z}{\partial x}\right.$ or $\left.-g h \frac{\partial Z}{\partial y}\right)$.

ii. Bed friction by the use of a Chezy's coefficient (small water depths) or a Strickler's coefficient.

Gravity terms are treated as fluxes in such a way as an horizontal water surface remains strictly horizontal (Paquier [21]). For instance, the integral on one cell $\iint-g h \frac{\partial Z}{\partial x} d x d y$ becomes

$$
0.5 \sum_{\text {edges }} \alpha_{i} \frac{L_{i}}{S}\left(h_{c}+h_{i}\right)\left(Z_{i}-Z_{c}\right)
$$

in which $\alpha_{i}$ is the x-co-ordinate of the normal to the edge $i, L_{i}$ is the length of the edge, $h_{c}$ and $Z_{c}$ are the water depth and the bed elevation at the centre of the cell respectively, $h_{i}$ and $Z_{i}$ are the water depth and the bed elevation at the middle of the edge $i$ respectively, $S$ is the area of the cell.

Bed friction terms are more simply assessed at the centre of the cell. Their computation uses an implicitation in time in order to avoid numerical instabilities when a rapid change in water depth or velocity occurs.

\section{Automatic differentiation}

\subsection{Generality}

Derivatives play a fundamental role in various areas of computational science including optimization, data assimilation, assessment of uncertainties, sensitivity analysis, etc (Buecker et al. [1], Castaings et al. [3], Corliss et al. [4], Gilbert and Lemaréchal [10]). A classical way to obtain these derivatives (from computer models) is the divided differences method. The rounding errors and cancelation are important drawbacks of this approximation method; furthermore, these errors and complexity increase when higher derivatives are

calculated. To overcome these problems we use AD tool (Souhar [30], Souhar and Faure [31], [32]). 
The AD, just like the divided differences, requires only the original program. In mathematics, it's a method to numerically compute the partial derivatives of a function $F$ specified by a computer program ( Buecker et al. [1], Carle and Fagan [2], Giering [8], Griewank [13], Hascoet and Pascual [14]). An AD tool (Carle and Fagan [2] or Hascoet and Pascual [14] for example) builds a new source program that computes the derivatives of $F$. The tool behaves like a compiler, which first understands the meaning of the original program, represents it in an internal form, performs analyses to get deepen understanding, and generates an object code. AD exploits the fact that any computer program that implements a vector function $Y=F(X)$ can be decomposed into a sequence of elementary assignments, any one of which may be trivially differentiated by a simple table lookup. These elementary partial derivatives are combined to form some derivative information of $F$. This process leads to exact numerical derivatives, because derivatives are computed analytically. In $\mathrm{AD}$ there are two methods to compute derivatives, the so-called tangent linear model that computes directional derivatives and is easy to implement on computer. The second method is the reverse model that computes the gradient of a function at one execution of the adjoint program. This latter program is relatively expensive and should therefore be optimized whenever possible.

Let $\mathcal{M}$ be a general nonlinear model, a mapping from $\mathbb{R}^{n}$ of the input variables $X=\left(x_{1}, \ldots, x_{n}\right)^{T}$ (initial conditions, topographic data, roughness coefficient, boundary conditions ... in hydraulic model cases) to $\mathbb{R}^{m}$ of the output variables $Y=\left(y_{1}, \ldots, y_{m}\right)^{T}$ (model state, objective function ...):

$$
\begin{aligned}
F: \mathbb{R}^{n} & \longrightarrow \mathbb{R}^{m} \\
X & \longmapsto Y=F(X)
\end{aligned}
$$

The vectors $X \in \mathbb{R}^{n}$ and $Y \in \mathbb{R}^{m}$ may be represented with respect to the ordinary unit basis vectors $\left\{\vec{e}_{i} ; i=1, \ldots, n\right\}$ and $\left\{\vec{f}_{k} ; k=1, \ldots, m\right\}$ as

$$
X=\sum_{i=1}^{n} x_{i} \vec{e}_{i}, \quad Y=\sum_{k=1}^{m} y_{k} \vec{f}_{k} .
$$

The entries $x_{i}$ and $y_{k}$ forming the vectors $X$ and $Y$ are called input independent and output dependent variables respectively. And all the other variables used in the program to evaluate $F(X)$ are called intermediate.

Consider a perturbation $d X$ of the input variables. Its effect on the outputs may be obtained via the linear approximation of the model $\mathcal{M}$ in terms of 
its Jacobian matrix, evaluated in the point $X$ according to

$$
d Y=\nabla F(X) \cdot d X
$$

Equation (4.1) is called the tangent linear model (Hascoet and Pascual [14], Inria [15]).

$$
\delta y_{k}=\sum_{i=1}^{n} \frac{\partial F_{k}(X)}{\partial x_{i}} \delta x_{i},
$$

where, $d X=\sum_{i=1}^{n} \delta x_{i} \vec{e}_{i}$ and $d Y=\sum_{k=1}^{m} \delta y_{k} \vec{f}_{k}$.

The cost (algorithmic complexity) for computing gradients of scalar-valued functions with either divided differences method or tangent linear model of $\mathrm{AD}$ grows linearly with the number of variables, whereas the so-called reverse model of $\mathrm{AD}$, an extremenlly efficient way, can compute such gradients at constant cost (Griewank [12]). Thus, if there are many input variables $\left(10^{6}-10^{8}\right.$, for large-scale shallow water applications), it becomes prohibitive to proceed directly as in (4.2), and for these large applications the reverse model is used, see (Castaings et al. [3], Gilbert [9], Griewank [12], Hascoet and Pascual [14], Inria [15], Rall [28]) for more details.

In this work we use Tapenade, an AD software tool, to compute automatic derivatives of a two-dimensional hydraulic model. This tool is developed at INRIA Sophia-Antipolis by Tropics team (Hascoet and Pascual [14], Inria $[15])$.

\subsection{Building and validation of AD models}

Derivation of models is not so easy, indeed a lot of difficulties can occur if direct models (the source models which must be differentiated) have not been written in the goal to be differentiated.

The first step in using Tapenade, in particular to derive Rubar20 model, is to load the input source program and to identify which routine implements the mathematical function that we want to differentiate. We should then share the model in two parts corresponding respectively to the reading of data and initialization of the model and to the calculation a long the time. Therefore only the part of the model that achieves the computations is going to be differentiated.

A pre-treatment step for AD strategy must be done. Tapenade may issue a 
number of error and warning messages when differentiating a file is done. For example, in the source of the direct model there exists some subroutines with subroutine as arguments. Tapenade doesn't accept this type of instructions and hence obliges the user to modify manually the source model.

A post-treatment step is also required, it consists to insert read data and write results instructions in the differentiated subroutines and the necessary calls of subroutines like those of initializations. After this, a part of debugging compilation errors must be done (Souhar [30]).

Tapenade can be utilzed as a server, which runs at INRIA Sophia-Antipolis at http://tapenade.inria.fr.8080/tapenade/indes.jsp. It can also be installed locally, in that case it is run by a simple command line, which can be included into a Makefile. Figure 1 shows a typical Makefile built to make easy the derivation of the code after each modification.

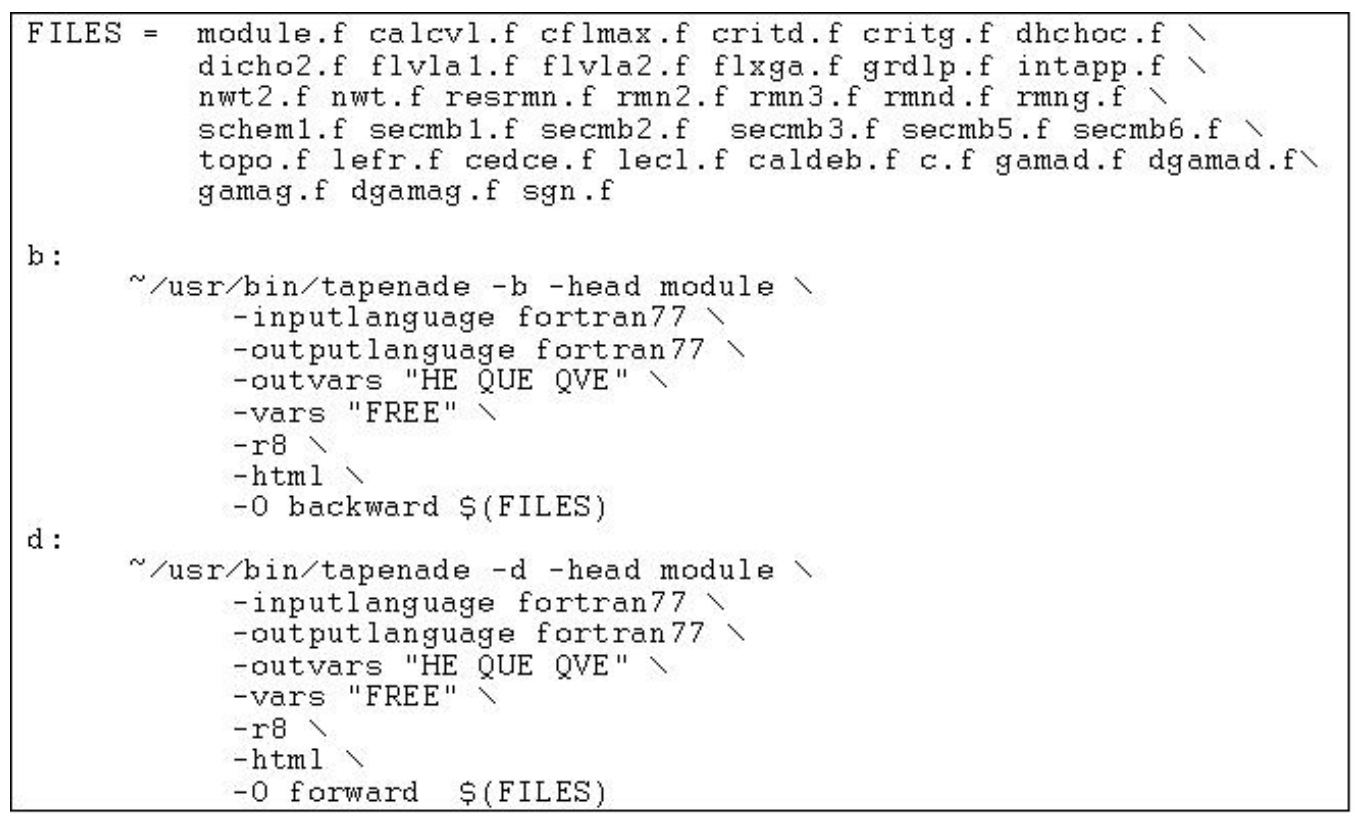

Figure 1: A typical Makefile of "Rubar20"

So the options are defined as follows.

- head allows to specify the top differentiation routine that implements the mathematical function. 
- inputlanguage allows to select the source code language.

- outputlanguage allows to select the output differentiated code language.

- outvars allows to define the output variables and whose derivatives are required (here the outputs are the water depth and discharge).

- vars allows to choose the input variables with respect to which differentiated must be made (here the inputs are the friction coefficients).

- $O$ point out the directory of the differentiated code.

The command make $d$ (resp. make b) permit then to obtain the tangent linear model (resp. the reverse model) of the source program defined by its arborescence given in FILES option of Fig. 1.

Tapenade will probably not produce a perfect differentiated program at first try. After AD, a validation step is necessary to check the computed derivatives. It should be noted that a common technique to check validation of these derivatives is to compare them to finite differences approximations, for example:

$$
\frac{\partial F(x)}{\partial x_{i}}=\lim _{\varepsilon \rightarrow 0} \frac{1}{\varepsilon}\left[F\left(x_{1}, \ldots, x_{i}+\varepsilon, \ldots, x_{n}\right)-F\left(x_{1}, \ldots, x_{i}, \ldots, x_{n}\right)\right]
$$

for $i=1, \ldots, n$. In (Hascoet and Pascual [14], Inria [15]), the developers of Tapenade proposed this technique to validate tangent linear models and it is called "gradient test". The so-called "dot product test" is proposed in the same user's guide to validate reverse models.

Figure 2 below shows the validation of derivatives of Rubar20 obtained by Tapenade. This figure shows well that the error function $r(\varepsilon) \longrightarrow 0$ when $\varepsilon \longrightarrow 0$, where $r$ is defined as follows:

$$
r(\varepsilon)=\left\|\frac{F(x+\varepsilon \cdot d)-F(x)}{\varepsilon}-\nabla F(x) \cdot d\right\|_{2},
$$

where $d=(1, \ldots, 1)^{T}$ is the unit vector of $\mathbb{R}^{n}$ and $\nabla F(x) . d$ is computed by Tapenade. 


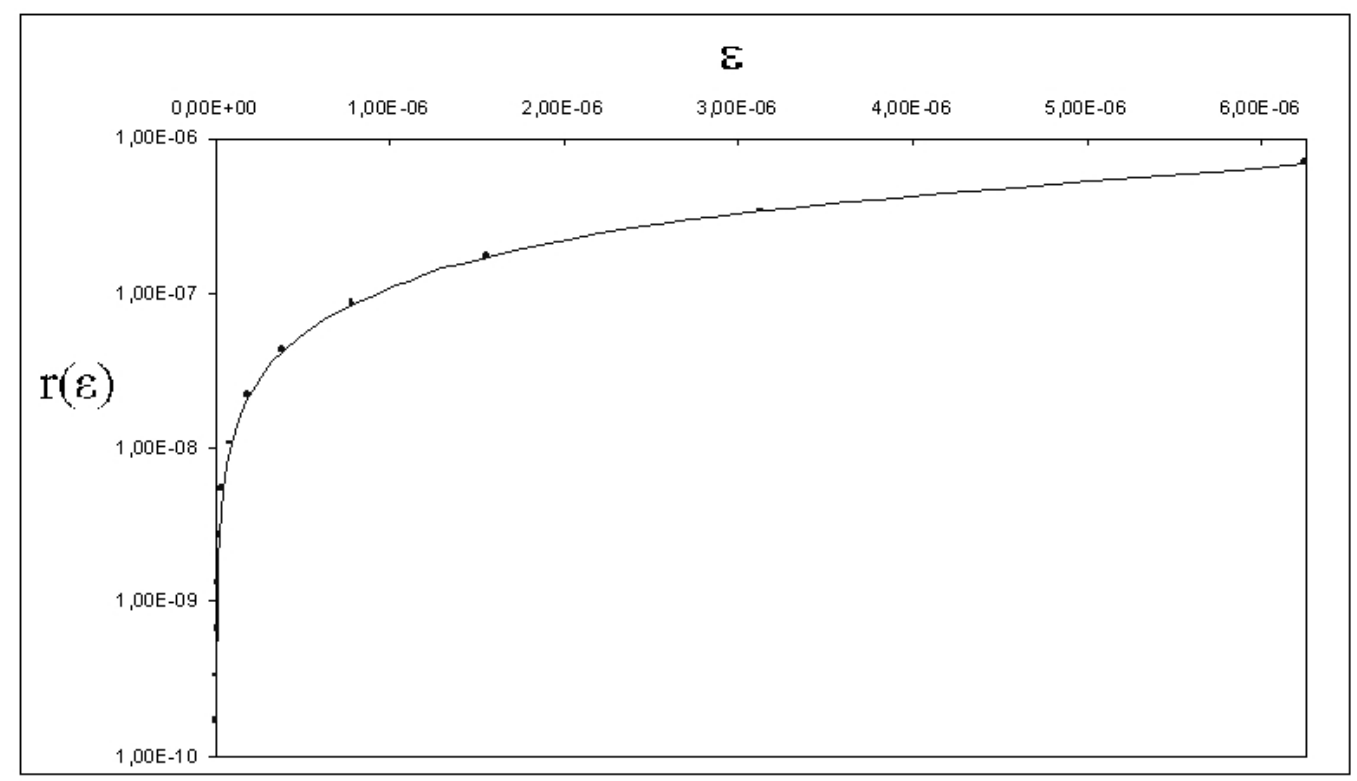

Figure 2: Gradient test for a 2-D model: "Rubar20"

\section{$5 \quad$ Numerical experiment}

In this Section, first we propose one example to show the robustness and validity of our numerical model Rubar20; secondly we demonstrate the efficiency of the forward sensitivity method, which uses AD-generated derivatives computed by Tapenade.

In all of our tests, the precision for water depths is $10^{-4} \mathrm{~m}$, the correction Van Leer's coefficient is 0.6 , the Courant number is 0.5 and the duration of the simulation is $80 \mathrm{~s}$.

\section{$5.1 \quad$ Validity of Rubar20}

We are interested here in a complex problem. The flow, a portion of a river of length $28.5 \mathrm{~m}$ and width $20 \mathrm{~m}$, is perturbed by some obstacles. The portion is crossed by a bridge with two triangles representing the bridge abutments and two cylindric bridge piers schematized by two octagons (see Fig. 3).

The cross sections of the main channel are trapezoidal of slope $2 / 1$, a discharge of $62 \mathrm{~m}^{3} / \mathrm{s}$ constitutes the upstream boundary condition, the downstream condition is imposed such that $Z+h=0$. The friction coefficient 
according to Manning law is homogeneous and uses a Strickler's coefficient equal to 40 .

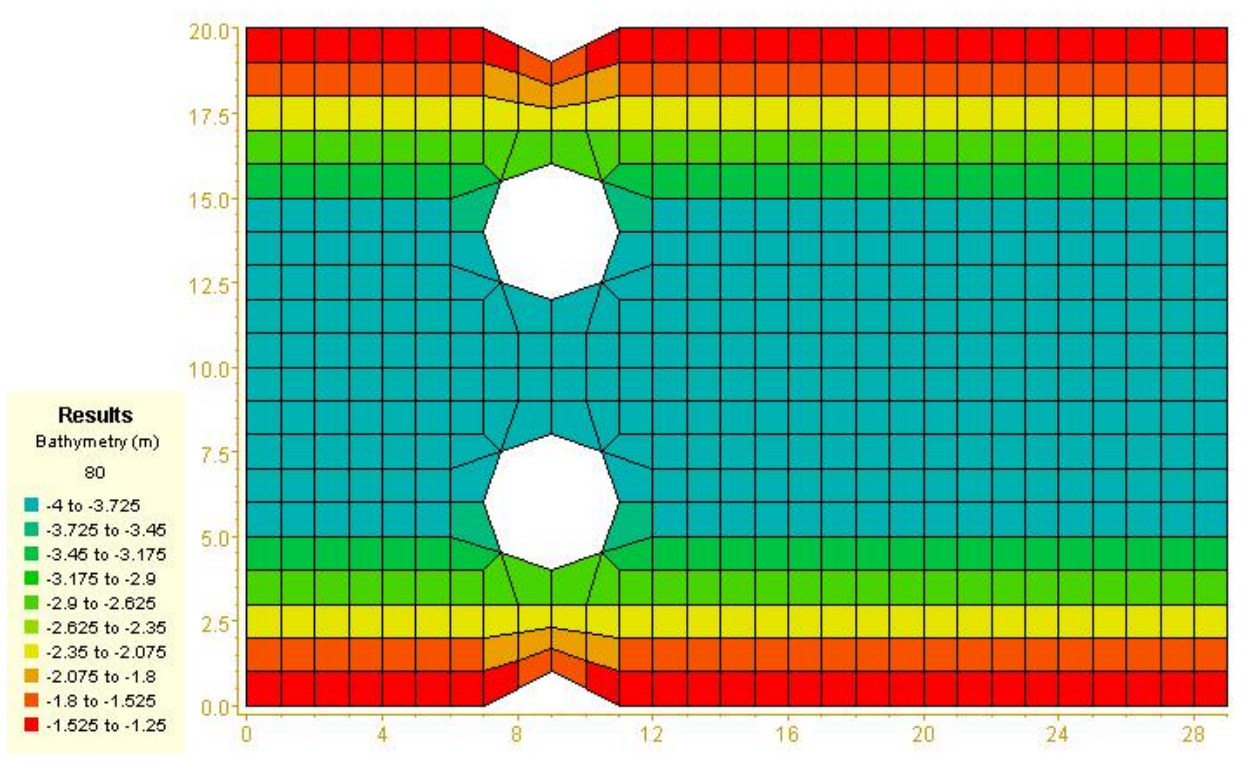

Figure 3: Grid for computation of the study

Fig. 4 and Fig. 5 provide water depths $(m)$ and discharge fields $\left(m^{2} / s\right)$ computed by Rubar20 for two diffusion coefficient values. According to these figures we remark that the model conserve the symmetry of the results because of the symmetry of the posed problem. We study now the recirculation behind the bridge piers and the bridge abutments for two different diffusion coefficients $D$. Fig. 4 and Fig. 5 give the velocity fields which permits to assess the recirculation's length. For $D=5 \cdot 10^{-2}$ (Fig. 4), the results are near to those computed by Télémac (Télémac is a numerical model developed by Electricité de France) (Galland et al. [7]). For $D=5 \cdot 10^{-3}$, Télémac finds eddies behind the bridge piers. Rubar20 gives recirculations behind the bridge abutments (Fig. 5). This difference results mainly from a small difference of grids used for computations in the two codes. 


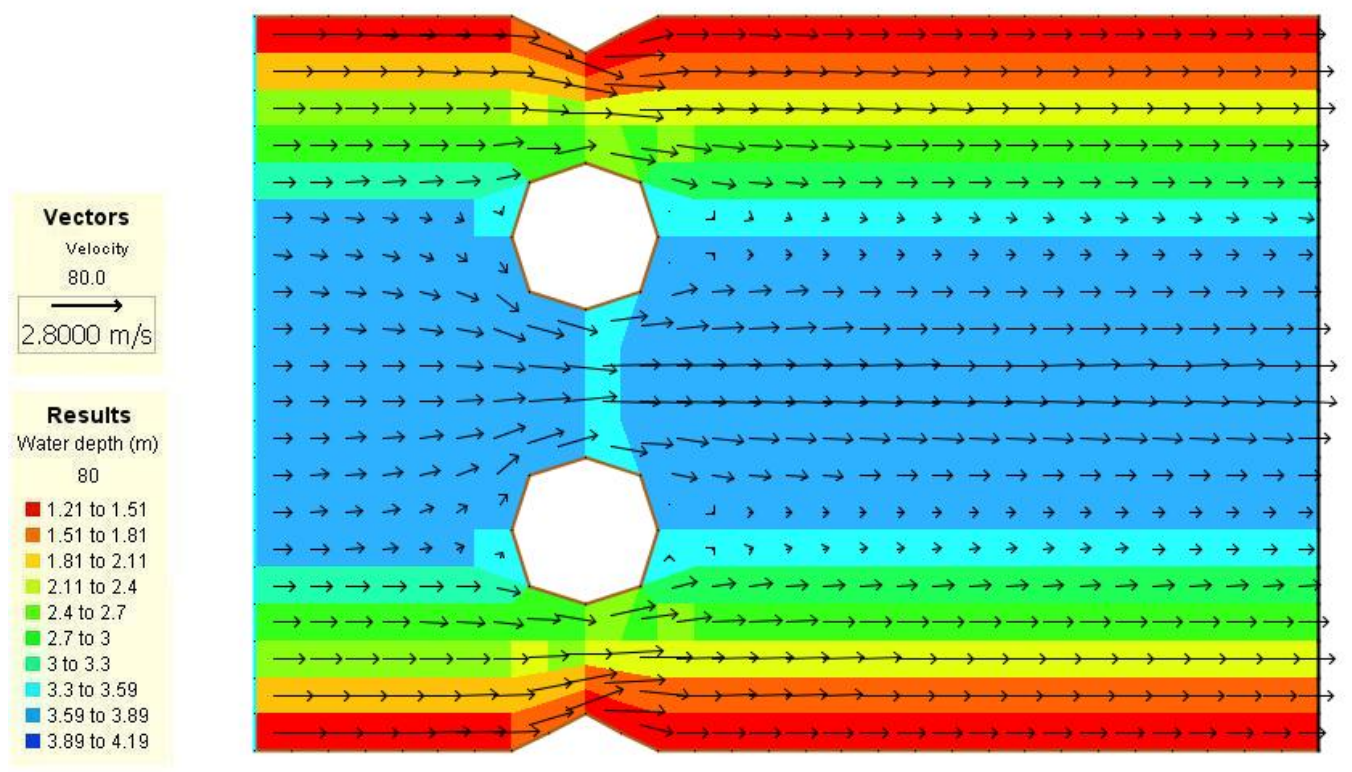

Figure 4: Velocity field in steady flow using Rubar20: $D=5 \cdot 10^{-2}$

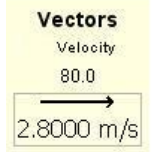

Results Water depth (m) 80

E 1.13 to 1.44 1.44 to 1.74 1.74 to 2.05

2.05 to 2.36

-2.36 to 2.66

2.97 to 3.27

$=3.27$ to 3.58

3.58 to 3.89

3.89 to 4.19

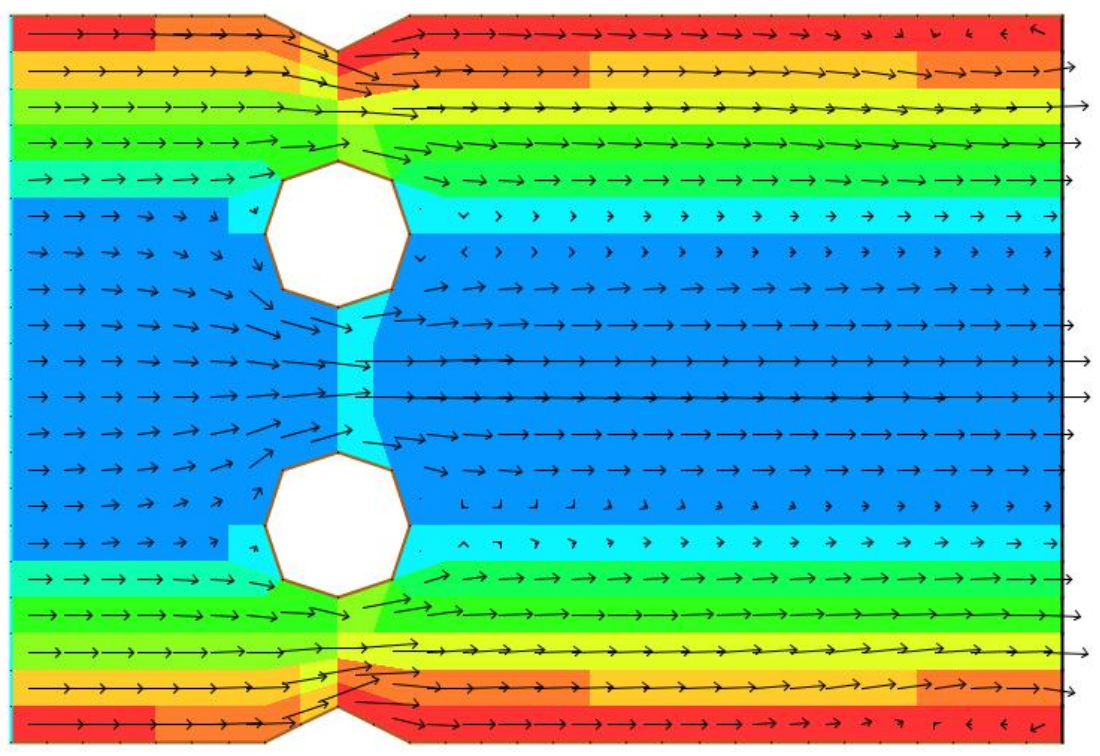

Figure 5: Velocity field in steady flow using Rubar20: $D=5 \cdot 10^{-3}$ 


\subsection{Sensitivity analysis}

Assessment and sensitivity analysis play an important role in decision making on flood control, so minimization of the risk should be the general objective. In the following we are interested in the sensitivities of water depth and discharge with respect to the roughness coefficient. We compute via $\mathrm{AD}$ the corresponding derivatives.

The study has a homogeneous roughness, then it is important to define the subspace $\mathbb{K}=\operatorname{span}\{\varphi\}$ of $\mathbb{R}^{n}$, where $n=548$ is the number of computational points and the basis vector is $\varphi=(1, \ldots, 1)^{T} \in \mathbb{R}^{n}$, in this case the function $F$ of Subsection 4.1 becomes:

$$
\begin{aligned}
F: \mathbb{K} & \longrightarrow \mathbb{R}^{m} \\
(S T K) & \longmapsto F(S T K)=\left(F_{1}(S T K), \ldots, F_{m}(S T K)\right)^{T},
\end{aligned}
$$

where $F_{j}(S T K)$ represents the water depth or the discharge at section $j$ and $m=548$, and $S T K$ is the friction coefficient.

According to Taylor's formula, which illustrates the physical definition of the gradient, the values of the function $F$ at the points $S T K$ and $S T K+\delta \varphi$ are related by:

$$
F(S T K+\delta \varphi)=F(S T K)+\delta \nabla F(S T K) \cdot \varphi+\mathcal{O}\left(\delta^{2} \varphi\right),
$$

with

$$
\nabla F(S T K)=\left(\begin{array}{c}
\frac{d F_{1}(S T K)}{d x} \\
\cdot \\
\cdot \\
\cdot \\
\frac{d F_{m}(S T K)}{d x}
\end{array}\right)
$$

Fig. 6 and Fig. 7 show gradients of water depths $(m)$ and discharges $\left(\mathrm{m}^{2} / \mathrm{s}\right)$ related to the friction coefficient (defined by (5.3)) computed using the AD tool Tapenade. So the sensitivities of the water depths and discharges are also symmetric for both diffusion coefficient values. We remark that these sensitivities vary in the opposite of the diffusion coefficient; i.e., sensitivities are less high for $D=5 \cdot 10^{-2}$ than for $D=5 \cdot 10^{-3}$. We remark also that discharge's sensitivities are high behind the bridge piers and the bridge abutments for both diffusion coefficient values. 


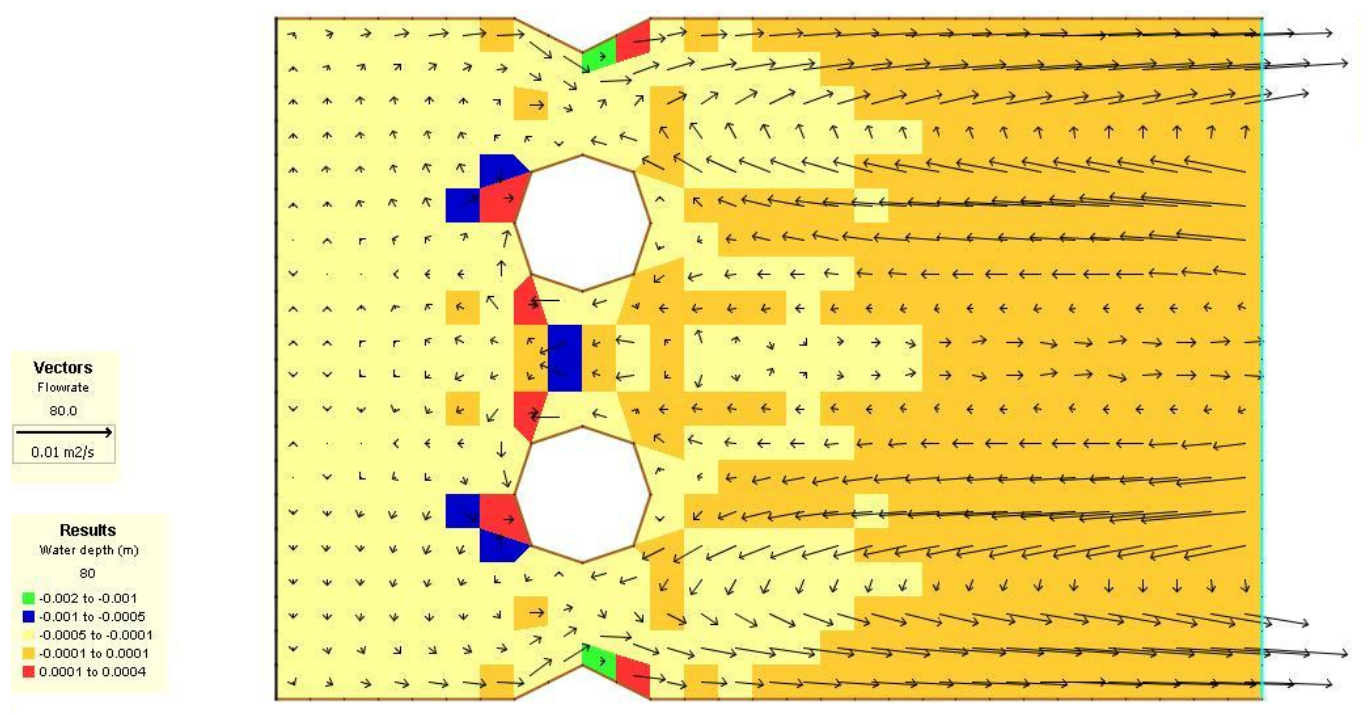

Figure 6: Sensitivities of water depths and discharges to bottom friction: $D=5 \cdot 10^{-2}$

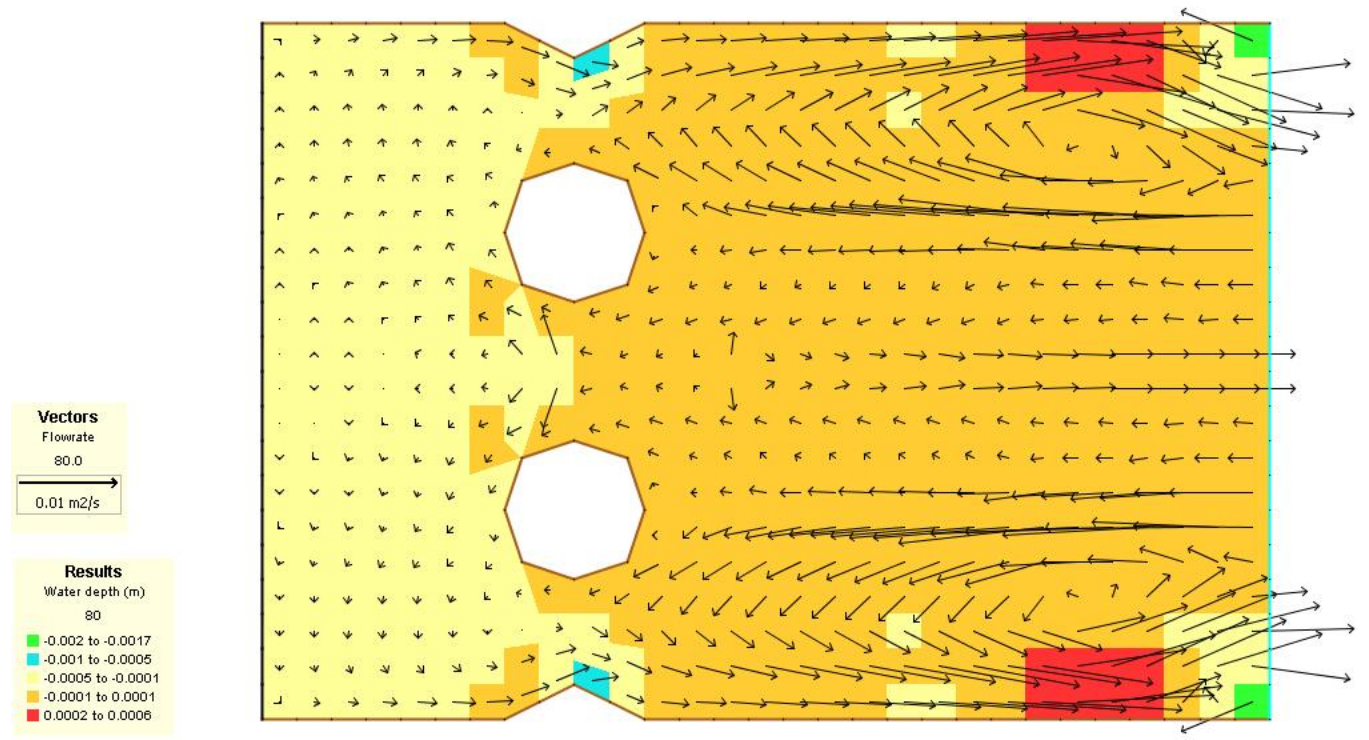

Figure 7: Sensitivities of water depths and discharges to bottom friction: $D=5 \cdot 10^{-3}$ 
According to Fig. 4 and Fig. $6\left(D=5 \cdot 10^{-2}\right)$, an increase of the friction coefficient may lead to find recirculations behind the bridge piers, whereas a decrease of the friction coefficient may lead to find recirculations behind the bridge abutments. From Fig. 5 and Fig. $7\left(D=5.10^{-3}\right)$, we remark that an increase of the friction coefficient may lead to find more important recirculations behind the bridge piers and to increase recirculations zones behind the bridge abutments.

\section{Conclusion and remarks}

The two-dimensional hydraulic model Rubar20 provides satisfactory results faced to a complex problem. The model can provide detailed velocity field and water levels. But a computation with fine grids should be used to detail and understand the recirculation problem more accurately. The computational technique can be easily extended to channels with arbitrary cross section, for computing the propagation of a flood surge on a dry bed, also for the computation of the flood wave resulting from the break of a dam, etc.

We have shown how AD tool can aid in the sensitivity analysis of a two dimensional finite volume model of shallow water flows. So the numerical sensitivities of water depths and discharges related to the friction coefficient are also symmetric as the results provided by the model and because of the symmetry of the channel. Furthermore, these sensitivities become more important for a small diffusion coefficient. Such sensitivities employed the forward derivatives computed using the AD software Tapenade. But it is still necessary to manually modify the source codes before automatic derivative step because AD tools still remain unable to take into account all programming instructions.

\section{References}

[1] Buecker, H-M., Corliss, G., Hovland, P., Naumann, U., Noriss, B. 2005 Automatic Differentiation: Applications, theory and tools, Springer, New York (NY), Lecture Notes in Computational Science and Engineering, Chapter 34, 293 - 298.

[2] Carle, A. and Fagan, M. 2000 ADIFOR 3.0 Overview, Rice University Report CAAM-TR-00-02. 
[3] Castaings, W., Dartus, D., Honnorat, M., Le Dimet, F.-X., LOUKILI, Y. AND MonNIER, J. 2004 Automatic differentiation: a tool for variational data assimilation and adjoint sensitivity analysis for flood modelling, AD 2004 - Fourth International Workshop on Automatic Differentiation, July 19-23, 2004, Argonne National Laboratory, Gleatcher Center,Chicago, IL, USA.

[4] Corliss, G., Faure, C.,Griewank, A., Hascoet, L., Naumann, U. 2001 Automatic Differentiation of Algorithms From Simulation to optimization, Springer, Computer and Information Science.

[5] Dhervillez, L. 2001 Incertitudes sur l'extension d'une zone inondable, DESS Thesis, Joseph Fourier University, Grenoble, France, 110 pages.

[6] Faure, J-B., Dhervillez, L. And Vidal, J-P. 2002 Automatic evaluation of the uncertainty on a flooded area, Hydroinformatics'2002, Cardiff, UK.

[7] Galland, J.C., Hervouet, J.M., Rouge, D. And Thellier, P. 1991 Code TELEMAC, Version 1.0, Note de validation, Electricité de France/Direction des études et Recherches HE-42/90.06.

[8] Giering, R. 1997 Tangent linear and Adjoint model compiler, Users manual, http://www.autodiff.com/tamc, FastOpt Gmbh.

[9] Gilbert, J. C. 1992 Automatic differentiation and iterative processes, Optimization methods and software, 1, 13-21.

[10] Gilbert, J. C., And Lemaréchal, C. 1989 Some numerical experiments with variable storage quasi-Newton algorithms, Mathematical Programming 45: 407 - 435.

[11] Giraud, F. M., Faure, J-B., Zimmer, D., Lefeuvre, J.C. And SkAGGS, R.W. 1997 Hydrologic modeling of a complex wetland, Journal of Irrigation and Drainage Engineering, 123: 5, 344-353.

[12] Griewank, A. 1993 Some bounds on the complexity of gradients, Jacobians, and Hessians, In Panos M. Pardalos, editor, Complexity in Nonlinear Optimization, 128-161, World Scientific Publishers. 
[13] Griewank, A. 2000 Evaluating Derivatives: Principales and Techniques of Algorithmic Differentiation, Number 19 in Frontiers in Applied Mathematics, SIAM, Philadelphia.

[14] Hascoet, L. and Pascual, V. 2004 Tapenade 2.1 user's guide, Rapport INRIA n 300, Septembre 2004, 78 pp. http://www.inria.fr/rrrt/rt0300.html

[15] InRIA, Projet TROPICS, TAPENADE tutorial. http://wwwsop.inria.fr/tropics/

[16] LAI, C. 1986 Numerical modelling of unsteady open-channel flow, Adv.in Hydroscience,14,162-323.

[17] Mignot, E. 2005 Etude expérimentale et numérique de l'inondation d'une zone urbanisée : cas des écoulements dans les carrefours en croix, Thèse en Mécanique, Ecole Centrale de Lyon.

[18] Mignot, E., Paquier, A. And Haider, S. 2006 Modeling floods in a dense urban area using $2 D$ shallow water equations, Journal of Hydrology, vol. 327, n 1-2, 186-199.

[19] Mignot, E., Paquier, A. And Ishigaki, T. 2006 Comparison of numerical and experimental simulations of a flood in a dense urban area, Water science and technology, vol. 54, n 6-7, p. 65-73.

[20] NaAim, M. 1991 Numerical modelling of hydrodynamic effects caused by a solid sliding in a reservoir, Thèse de l'université Joseph Fourier Grenoble 1.

[21] Paquier, A. 1995 Modélisation et simulation de la propagation de l'onde de rupture de barrage, Thèse de l'université de Saint-Etienne.

[22] Paquier, A. 1998 1-D and 2-D models for simulating dam-break waves and natural floods, Union Européenne CADAM meeting, Wallingford, GBR, Mars 1998.

[23] Paquier, A. 2003 Surface flows during high floods in towns, Houille blanche, vol. 6, p. 89-93.

[24] Paquier, A. and Farissier, P. 1996 Use of a 2-D model fo simulating the flooding of a plain, Hydroinformatics'96, Muller, 129-136. 
[25] Paquier, A. And Mignot, E. 2003 Use of 2D models to calculate flood water levels: calibration and sensitivity analysis, XXX IAHR congress, Thessaloniki, GRC, 24-29 August 2003.

[26] Paquier, A., Tanguy, J.M., Haider, S. And Zhang, B. 2003 Estimation of the flood levels for a flash flood in urban area: comparison of two hydrodynamic models on the 1988's Nimes flood, Revue des sciences de l'eau, vol. 16, n 1, p. 79-102.

[27] Proust, S., Rivière, N., Bousmar, D., Paquier, A., Zech, Y. AND MOREL, R. 2006 Flow in compound channel with abrupt floodplain contraction, Journal of hydraulic engineering, vol. 132, n 9, p. 958-970.

[28] Rall, L. B. 1981 Automatic Differentiation Techniques and Applications, Lecture Notes in Computer Science 120 Springer-Verlag, Berlin.

[29] Roe, P. L. 1981 Approximate Riemann solvers, parameter vectors and difference schemes, Journal of Computational Physics 43:357-372

[30] Souhar, O. 2006 Evaluation of uncertainties for 1-D fluvial model. Automatic differentiation of 2-D Shallow water flows by Tapenade, Tech. Rep., Cemagref de Lyon, CHHLY.20108 (LY), 31 pp.

[31] Souhar, O. And Faure, J-B. 2006 Two gradient techniques to study sensitivities applied to 1-D and 2-D hydraulic models, Submitted.

[32] Souhar, O. AND FAure, J-B. 2007 Uncertainty propagation and sensitivity derivatives of a hydraulic model via automatic differentiation, Submitted. 